\title{
Power-Efficient Dynamic Quantization for Multisensor HMM State Estimation over Fading Channels
}

\author{
Nader Ghasemi and Subhrakanti Dey \\ Department of Electrical and Electronic Engineering \\ University of Melbourne \\ Parkville, Melbourne, VIC 3010 Australia \\ e-mail: \{n.ghasemi, s.dey\}@ee.unimelb.edu.au
}

\begin{abstract}
In this paper, we address the problem of designing power efficient quantizers for state estimation of hidden Markov models using multiple sensors communicating to a fusion centre via error-prone randomly time-varying flat fading channels modelled by finite state Markov chains. Our objective is to minimize a tradeoff between the long term average of mean square estimation error and expected total power consumption. We formulate the problem as a stochastic control problem by using Markov decision processes. Under some mild assumption on the measurement noise at the sensors, the discretized action space (quantization thresholds and transmission power levels) version of the optimization problem forms a unichain Markov decision process for stationary policies. The solution to the discretized problem provides optimal quantization thresholds and power levels to be communicated back to the sensors via a feedback channel. Moreover, in order to improve the performance of the quantization system, we employ a gradientfree stochastic optimization technique to determine the optimal set of quantization thresholds from which optimal quantization levels are determined. The performance results for estimation error/total transmission power tradeoff are studied under various channel conditions and sensor measurement qualities.
\end{abstract}

Index Terms-Power control, state estimation, hidden Markov models, fading channels, Markov decision processes.

\section{INTRODUCTION}

In recent years, due to the wide range of applications of wireless sensor networks (WSNs), signal processing with distributed sensors has gained great research interest especially in context of distributed detection and estimation. A typical wireless sensor network (WSN) consists of a set of geographically dispersed sensor nodes which have limited battery power and communication capability. Due to energy limitations and bandwidth constraints encountered with WSNs, the transmission data rate of each individual sensor node is severely limited. This motivates the requirement for quantizing observations at sensor nodes so that only a short sequence of bits representing the observation is transmitted to the fusion centre.

Designing such quantizers optimally is quite challenging. However, many authors have obtained iterative quantizer design methods under various different distortion criteria for distributed detection (hypothesis testing) as well as for decentralized estimation of random variables with known densities (e.g., [1]). Quantizer design for decentralized parameter estimation

This work was partially supported by the Australian Research Council.

N. Ghasemi and S. Dey are with the ARC Special Research Centre on Ultra-Broadband Information Networks (CUBIN), Department of Electrical and Electronic Engineering, University of Melbourne, Parkville, Melbourne, VIC 3010 Australia. CUBIN is affiliated program of National ICT Australia (NICTA). was considered in [2] using multiple sensors with Bayes distortion and Fisher information criteria. There is also an extensive literature on binary sensors in the context of binary hypothesis testing; see [3]-[5] and references therein. Various decentralized/distributed parameter estimation problems with quantized (binary or with a small number of bits) data have been studied in [6]-[8] (see also references therein).

In contrast, the design of optimal quantizers for decentralized state/parameter estimation of dynamical systems, to minimize estimation error variance, is a difficult task because this error variance can be a complex nonlinear nonconvex function of the quantization thresholds. Recently, a stochastic control approach has been used in [5] to design optimal quantizers for general hidden Markov models (HMMs). These results, however, do not take into account other constraints in WSNs such as limited energy/memory/computing power, sensor faults, channel noise or random channel conditions such as fading etc. that can lead to signal degradation. Some of these constraints have been addressed partially in recent works such as [9] for constant parameter estimation with energy constraints, while the impact of channel noise and fading is considered in [10] for distributed detection. While [5] assumed error-free communication between sensors and the fusion centre, a more recent study [11] extends these works by considering more realistic case of additive channel noise and wireless channel fading. In [11] the wireless fading channel is modeled by a finite-state Markov chain.

In most application scenarios of WSNs, sensor nodes are powered by small batteries for which replacement is impractical, if not impossible, due to cost limitations or because they are deployed in hostile environments with high temperature, high pollution levels, and so on. This highly motivates the fact that power-efficient designs are of chief importance [12]. Minimization of transmission power leads to more efficient utilization of battery energy and hence longer battery life of sensor nodes. On the other hand, reducing transmission power increases the probability of error as a result of transmission over a noisy fading channel, thus increasing the average state estimation error at the fusion centre. Motivated by these concerns, in this paper, we address the issue of minimizing the average state estimation error (at the fusion centre) with respect to sensor quantization thresholds and sensor transmission power levels, under a fixed expected total transmission power constraint. In particular, we study a decentralized state estimation problem for a remote stochastic process (such as target maneuvers in battlefield scenarios, or gas/chemical leaks in hostile environments etc.) modelled by an underlying finite state discrete time Markov chain via multiple sensors. We con- 
sider the sensors to have modest computation capability, in that they only quantize their measurements into a binary symbol. We formulate the problem using a Markov decision process (MDP) approach, namely, an unconstrained Lagrangian based MDP formulation. Our work is distinct from [11] in that we allow different sensors to be allocated different power levels while bounding the total expected power allowed per time of operation. Moreover, we introduce a framework to determine the set of quantization thresholds optimally rather than arbitrarily. This leads to a significant improvement in optimizing the estimation error/total power tradeoff cost. We account for the error-prone randomly time-varying flat fading channel between each sensor and the fusion centre by modeling it as a binary symmetric channel with the crossover probability controlled by the transmission power and channel status.

The remainder of the paper is outlined as follows: in Section II, we describe a model for the system and present the hidden Markov model formulation of the state estimation problem using quantized measurements with power constraint. In Section III, we present the MDP formulation of optimizing the tradeoff between average estimation error and average power consumption across the sensors for the presented model. In Section IV, we describe a framework to determine the optimal set of quantization thresholds. The simulation results are given in Section $\mathrm{V}$ followed by some concluding remarks in Section VI.

\section{Problem Statement}

\section{A. System Model}

Let the dynamic process be represented by the random sequence $\left\{X_{t}, t \geq 1\right\}$ which is a stationary ergodic Markov chain with state space $\mathcal{X}=\left\{s_{1}, \cdots, s_{n}\right\}$. For this Markov chain, the transition probability is given by the matrix $\mathbf{P}=\left[p_{i j}^{\mathcal{X}}\right]$, where $p_{i j}^{\mathcal{X}}=\operatorname{Pr}\left(X_{t+1}=s_{j} \mid X_{t}=s_{i}\right), 1 \leq$ $i, j \leq n$. The state variable $X_{t}$ is related to the measurements by $\mathbf{Y}_{t}=\mathbf{A} X_{t}+\mathbf{V}_{t}$, where $\mathbf{Y}_{t}=\left[Y_{1, t}, \cdots, Y_{M, t}\right]^{T}$, $\mathbf{A}=[1, \cdots, 1]^{T}$, and the additive noise sequence $\mathbf{V}_{t}=$ $\left[\mathcal{V}_{1, t}, \cdots, \mathcal{V}_{M, t}\right]^{T}$ is independent and identically distributed (i.i.d.). The joint probability density function for $\mathbf{V}_{t}$ is given by $f_{\mathbf{V}}$.

We consider a one-bit binary quantization scheme in which the sequence $\left\{\mathbf{r}_{t}, t \geq 1\right\}=\left\{\left(r_{1, t}, \cdots, r_{M, t}\right), t \geq 1\right\}$ represents the sequence of quantization parameters. Let the quantized data be denoted by $\mathbf{Y}_{t}^{q}=\left[Y_{1, t}^{q}, \cdots, Y_{m, t}^{q}\right]^{T}$. Each sensor transmits its quantized output to the fusion centre over a discrete time flat fading channel. Let $\mathbf{C}_{t}=\left(C_{1, t}, \cdots, C_{M, t}\right)^{T}$ be the sensors' channel state vector at time $t$. We assume that the sequence of channel states $\left\{C_{m, t}, t \geq 1\right\}$ is a stationary ergodic Markov chain with state space $\mathcal{C}=\left\{c_{1}, \cdots, c_{k}\right\}$ and transition probability matrix $\mathbf{P}_{m}^{\mathcal{C}}=\left[p_{i j}^{m}\right]$, where

$$
p_{i j}^{m}=\operatorname{Pr}\left(C_{m, t+1}=c_{j} \mid C_{m, t}=c_{i}\right), \quad \begin{gathered}
1 \leq i, j \leq k \\
1 \leq m \leq M
\end{gathered} .
$$

Each channel state $c_{i}, 1 \leq i \leq k$ may represent a value of the channel gain. In the estimation problem, the channel states of different sensors are assumed to be known at the fusion centre, but not at the sensor nodes.

The transmission power at each sensor is allowed to vary according to the channel condition. Denote the $m$-th sensor's transmission power at time $t$ by $p_{m, t}$. Let $\mathbf{p}_{t}=$ $\left(p_{1, t}, \cdots, p_{M, t}\right)$ and write the sequence $\left\{\mathbf{p}_{t}, t \geq 1\right\}=$ $\left\{\left(p_{1, t}, \cdots, p_{M, t}\right), t \geq 1\right\} \cdot \mathbf{p}_{t}$ will be called power level parameter. Transmission power for every sensor is chosen from the same set of available power levels $\mathcal{P}=\left\{p_{1}, \cdots, p_{\ell}\right\}$
The received symbol at the fusion centre after decoding is then denoted by $Y_{m, t}^{f}$. Let $\mathbf{Y}_{t}^{f}=\left[Y_{1, t}^{f}, \cdots, Y_{M, t}^{f}\right]^{T} . Y_{m, t}^{f}$ is described by the following channel transition probability

$$
\operatorname{Pr}\left(Y_{m, t}^{f}=a_{j} \mid Y_{m, t}^{q}=a_{i}, C_{m, t}=c, p_{m, t}=p\right)=q_{i j}^{m}(c, p)
$$

where $i, j \in\{1,2\}, c \in \mathcal{C}, p \in \mathcal{P}$, and $a_{1}, a_{2}$ denote the symbols for a binary-quantized measurement.

The resulting input-output transition matrix is denoted by $\mathbf{Q}^{m}(c, p)=\left[q_{i j}^{m}(c, p)\right]$ conditioned on the channel state $c \in \mathcal{C}$ and power level $p \in \mathcal{P}$. The off-diagonal entries in $\mathbf{Q}^{m}(c, p)$ are called crossover probabilities. The crossover probabilities are formulated in the next section.

For each pair of sequences $\mathbf{r}=\left\{\mathbf{r}_{t}, t \geq 1\right\}$ and $\mathbf{p}=$ $\left\{\mathbf{p}_{t}, t \geq 1\right\}$, the long term average of the mean square error in state estimation is given by

$$
J(\mathbf{r}, \mathbf{p}):=\limsup _{T \rightarrow \infty} \frac{1}{T} \sum_{t=1}^{T} \mathbb{E}\left|X_{t}-\widehat{X}_{t}\right|^{2}
$$

where the state estimate $\widehat{X}_{t}$ is a Borel measurable function of the sequence $\left\{\mathbf{Y}_{l}^{f}, \mathbf{C}_{l}, l \leq t\right\}$. The objective of this paper is to solve the dynamic quantization optimization problem which is to obtain the optimal sequences $\mathbf{r}$ and $\mathbf{p}$ such that the mean square estimation error $J(\mathbf{r}, \mathbf{p})$ is minimized constrained on the long term average of total power consumption across all the sensors. The optimization problem can be formulated as

$$
\begin{array}{rl}
\min _{\mathbf{r}, \mathbf{p}} & J(\mathbf{r}, \mathbf{p}) \\
\text { subject to : } & \limsup _{T \rightarrow \infty} \frac{1}{T} \sum_{t=1}^{T} \mathbb{E} \mathcal{Q}\left(\mathbf{C}_{t}, \mathbf{p}_{t}\right) \leq P_{\text {avg }}
\end{array}
$$

where $\mathcal{Q}\left(\mathbf{C}_{t}, \mathbf{p}_{t}\right)$ is a power consumption cost function defined in (14) (on page 3). Notice that the fusion centre can not find the optimal quantization scheme using the optimization problem (4) by directly minimizing the cost function $J(\mathbf{r}, \mathbf{p})$. This is because it has no exact knowledge on $X_{t}$. Therefore, in the following section, we define the information state $\theta_{t}$, which can be computed at the fusion centre using a recursive formula, and then in Section III we restate the definition of the cost function $J(\mathbf{r}, \mathbf{p})$ based on the information state, thus converting the partially observed problem to a fully observed stochastic control problem.

\section{B. The information state}

Define the information state vector $\theta_{t}=\left[\theta_{1, t}, \cdots, \theta_{n, t}\right]^{T}$, where $\theta_{i, t}=\operatorname{Pr}\left(X_{t}=s_{i} \mid \mathcal{D}_{t}\right), i \in\{1, \cdots, n\}, t \geq 1$ and $\mathcal{D}_{t}:=\mathcal{D}\left(\mathbf{Y}_{l}^{f}, \mathbf{C}_{l}, l \leq t\right)$ is the $\sigma$-algebra generated by the observations up to time $t$. Denote also

$$
H\left(s_{i}, \mathbf{r}_{t}, \mathbf{y}_{t}^{q}\right)=\operatorname{Pr}\left(\mathbf{Y}_{t}^{q}=\mathbf{y}_{t}^{q} \mid X_{t}=s_{i}, \mathbf{r}_{t}\right), i \in\{1, \cdots, n\}
$$

where $\mathbf{y}_{t}^{q}$ denotes a value for the random variable $\mathbf{Y}_{t}^{q}$, and we have

$$
H\left(s_{i}, \mathbf{r}, \mathbf{a}\right)=\int_{\mathcal{U}(\mathbf{r}, \mathbf{a})} f_{\mathbf{V}}\left(y_{1}-s_{i}, \cdots, y_{M}-s_{i}\right) d y_{1} \cdots d y_{M}
$$

where $\mathbf{a}=\left(a_{i_{1}}, \cdots, a_{i_{M}}\right), i_{m} \in\{1,2\}$ for $m=1, \cdots, M$, and the integration region $\mathcal{U}(\mathbf{r}, \mathbf{a}):=\left\{\mathbf{y} \in \mathbb{R}^{M} \mid \mathcal{G}(\mathbf{r}, \mathbf{y})=\right.$ a $\}$, in which $\mathcal{G}: \mathbb{R}^{M} \times \mathbb{R}^{M} \mapsto\left\{a_{1}, a_{2}\right\}^{M}$ is the binary 
quantization mapping. Based on the channel input-output transition probability defined in (2), we have

$$
\begin{array}{r}
Q\left(\mathbf{y}_{t}^{f}, \mathbf{y}_{t}^{q}, \mathbf{c}_{t}, \mathbf{p}_{t}\right)=\operatorname{Pr}\left(\mathbf{Y}_{t}^{f}=\mathbf{y}_{t}^{f} \mid \mathbf{Y}_{t}^{q}=\mathbf{y}_{t}^{q}, \mathbf{C}_{t}=\mathbf{c}_{t}, \mathbf{p}_{t}\right) \\
=\prod_{m=1}^{M} q_{i_{m} j_{m}}^{m}\left(c^{m}, p_{m, t}\right)
\end{array}
$$

where $\mathbf{c}_{t}=\left[c^{1}, \cdots, c^{M}\right]^{T}, c^{m} \in \mathcal{C}, \mathbf{y}_{t}^{q}$ and $\mathbf{y}_{t}^{f}$ denote values for random variables $\mathbf{Y}_{t}^{q}$ and $\mathbf{Y}_{t}^{f}$ respectively, in which $y_{m, t}^{q}=a_{i_{m}}$ and $y_{m, t}^{f}=a_{j_{m}}$ for $i_{m}, j_{m} \in\{1,2\}$. Assuming the channels from the sensors to the fusion centre to be independent, we define the product of $M$ channel transition probabilities as $p\left(\mathbf{C}_{t+1}=\mathbf{c}_{t+1} \mid \mathbf{C}_{t}=\mathbf{c}_{t}\right)=\prod_{m=1}^{M} p_{i_{m} j_{m}}^{m}$, where $c_{m, t}=c_{i_{m}}, c_{m, t+1}=c_{j_{m}}$ for $i_{m}, j_{m} \in\{1, \cdots, k\}$, and $1 \leq m \leq M$. Define the function

$$
\begin{aligned}
\psi_{i}\left(s_{i}, \mathbf{r}_{t}, \mathbf{p}_{t}, \mathbf{Y}_{t}^{f}=\mathbf{y}_{t}^{f}, \mathbf{C}_{t}=\mathbf{c}_{t}\right) \\
\quad=\sum_{\mathbf{y}_{t}^{q} \in\left\{a_{1}, a_{2}\right\}^{M}} H\left(s_{i}, \mathbf{r}_{t}, \mathbf{y}_{t}^{q}\right) Q\left(\mathbf{y}_{t}^{f}, \mathbf{y}_{t}^{q}, \mathbf{c}_{t}, \mathbf{p}_{t}\right)
\end{aligned}
$$

We also introduce the diagonal matrix

$$
\begin{aligned}
& \mathbf{\Psi}\left(s_{1}, \cdots, s_{n}, \mathbf{r}_{t}, \mathbf{p}_{t}, \mathbf{Y}_{t}^{f}=\mathbf{y}_{t}^{f}, \mathbf{C}_{t}=\mathbf{c}_{t}\right)= \\
& \left(\begin{array}{ccc}
\psi_{1}\left(s_{1}, \mathbf{r}_{t}, \mathbf{p}_{t}, \mathbf{y}_{t}^{f}, \mathbf{c}_{t}\right) & \\
& \ddots & \\
& & \psi_{n}\left(s_{n}, \mathbf{r}_{t}, \mathbf{p}_{t}, \mathbf{y}_{t}^{f}, \mathbf{c}_{t}\right)
\end{array}\right)
\end{aligned}
$$

Proposition 2.1: The recursion for the information state $\theta_{t}$ is given by

$$
\begin{aligned}
\theta_{t} & =\frac{p\left(\mathbf{c}_{t} \mid \mathbf{c}_{t-1}\right)}{\xi_{t}^{\prime}} \boldsymbol{\Psi}\left(s_{1}, \cdots, s_{n}, \mathbf{r}_{t}, \mathbf{p}_{t}, \mathbf{y}_{t}^{f}, \mathbf{c}_{t}\right) \mathbf{P}^{T} \theta_{t-1} \\
& :=\frac{1}{\xi_{t}} \boldsymbol{\Lambda}\left(\mathbf{r}_{t}, \mathbf{p}_{t}, \mathbf{y}_{t}^{f}, \mathbf{c}_{t}\right) \theta_{t-1}
\end{aligned}
$$

where $\mathbf{P}$ is the transition matrix of $\left\{X_{t}\right\}, \xi_{t}^{\prime}$ and $\xi_{t}$ are normalizing factors such that $\left\|\theta_{t}\right\|_{1}=1$.

The proof is standard and can be obtained by use of the Bayesian rule and the detail is omitted here $\xi_{t}=\left\|\boldsymbol{\Lambda}\left(\mathbf{r}_{t}, \mathbf{p}_{t}, \mathbf{y}_{t}^{f}, \mathbf{c}_{t}\right) \theta_{t-1}\right\|_{1}$ is a normalization factor.

\section{Channel crossover probabilities}

In this section, we formulate the channel input-output transition probabilities $q_{i j}^{m}(c, p)$ as defined in (2). The received signal at the fusion centre from the $m$-th sensor at time $t$ is given by $y_{m, t}^{r}=g_{m, t} \sqrt{p_{m, t}} b_{m, t}+\nu_{m, t}$, where $g_{m, t}, p_{m, t}$ are the channel gain and transmission power respectively, $b_{m, t} \in\{-1,1\}$ denotes the source bit and $\nu_{m, t}$ is the additive white Gaussian channel noise. Here $b_{t}=\left[b_{1, t}, \cdots, b_{M, t}\right]^{T}$ is generated in an obvious manner via binary quantization, defined by the mapping $\mathcal{G}\left(\mathbf{r}_{t}, \mathbf{y}_{t}\right)$, of the noisy sensor observation $\mathbf{Y}_{t}=\mathbf{y}_{t}$ for the state value $X_{t}=x_{t}, x_{t} \in \mathcal{X}$. Let the covariance of $\nu_{m, t}$ be denoted by $\sigma_{\nu}^{2}$. For this signal model, the signal-to-noise ratio (SNR) at time $t$ is given by $\gamma=g_{m, t} p_{m, t} / \sigma_{\nu}^{2}$. Let $\kappa$ denote the path loss exponent of the wireless channel, $d_{m}$ the distance between the $m$-th sensor and the fusion centre, and suppose binary phase shift keying (BPSK) modulation is used to transmit the sensor bits, then we can express the crossover probability after detection at the fusion centre as

$$
\varepsilon^{m}(\gamma)=Q\left(\sqrt{2 \alpha \gamma d_{m}^{-\kappa}}\right)=\int_{\sqrt{2 \alpha \gamma d_{m}^{-\kappa}}}^{\infty} \frac{1}{\sqrt{2 \pi}} e^{-\frac{t^{2}}{2}} d t
$$

where $\alpha$ is a constant. The input-output transition relationship between the fusion centre decoded output $Y_{m, t}^{f}$ and the transmitted bit $b_{m, t}$ is then expressed by $\operatorname{Pr}\left(Y_{m, t}^{f}=-b_{m, t} \mid b_{m, t}\right)=\varepsilon^{m}(\gamma)$. Since we consider the channel as a binary symmetric channel, the other crossover probability is also given by the same quantity.

\section{The Stochastic Optimal Control Problem}

\section{A. Average Estimation Error/Average Total Power Trade-off}

In the following, we present the two per-stage cost functions, i.e., expected state estimation error and expected total power consumption across the sensors. Given the observations $\mathcal{D}_{t}$, the state estimate $\widehat{X}_{t}$ can be written as

$$
\widehat{X}_{t}\left(\theta_{t}\right)=\mathbb{E}_{X}\left[X_{t} \mid \mathcal{D}_{t}\right]=\sum_{i=1}^{n} s_{i} \theta_{i, t} .
$$

We introduce the conditional cost function

$$
\begin{aligned}
\phi\left(\theta_{t}\right) & =\mathbb{E}_{X}\left[\left|X_{t}-\widehat{X}_{t}\right|^{2} \mid \mathcal{D}_{t}\right] \\
& =\sum_{i=1}^{n}\left[s_{i}-\sum_{j=1}^{n} s_{j} \theta_{j, t}\right]^{2} \theta_{i, t}
\end{aligned}
$$

which is computed using (12) in terms of $\theta_{t}$. Notice that the fusion centre can minimize the error cost in (13), since $\theta_{t}$ can be computed using recursion given in (10) having the observations $\mathbf{y}_{l}^{f}, \mathbf{c}_{l}, l \leq t$. It is obvious that the state space for the information state $\theta$ can be defined as the simplex $\mathcal{T}_{\theta}=\left\{\Omega \in \mathbb{R}_{+}^{n} \mid\|\Omega\|_{1}=1\right\} \subset \mathbb{R}^{n}$.

Define the average total power cost function $\mathcal{Q}\left(\mathbf{C}_{t}, \mathbf{p}_{t}\right)$ as

$$
\mathcal{Q}\left(\mathbf{C}_{t}=\mathbf{u}, \mathbf{p}_{t}=\mathbf{v}\right):=\sum_{m=1}^{M} v_{m} \pi_{u_{m}}^{m}
$$

where $\quad \mathbf{u}=\left[u_{1}, \cdots, u_{M}\right]^{T}, \quad u_{m} \in \mathcal{C}, \quad \mathbf{v}=\left[v_{1}, \cdots, v_{M}\right]^{T}$, $v_{m} \in \mathcal{P}$ for $1 \leq m \leq M$ are the values for the channel state and transmission power vectors at time $t$, and $\pi^{m}=$ $\left[\pi_{c_{1}}^{m}, \cdots, \pi_{c_{k}}^{m}\right]^{T}$ denotes the steady-state distribution of the channel state sequence $\left\{C_{m, t}, t \geq 1\right\}$ for the $m$-th sensor (note that by the previous assumptions in Section II-A such a distribution exists and is unique).

Due to the randomized nature of optimal solutions to constrained MDP problems, we now obtain an unconstrained MDP formulation, by minimizing the long-term average of a weighted combination of these two conflicting objectivesthe average estimation error $\phi\left(\theta_{t}\right)$ and average total power consumption cost $\mathcal{Q}\left(\mathbf{C}_{t}, \mathbf{p}_{t}\right)$. Thus, a variation of the optimal estimation problem (4) subject to the long run average total power consumption may be expressed as

$$
\min _{\mathbf{r}, \mathbf{p}} \limsup _{T \rightarrow \infty} \frac{1}{T} \sum_{t=1}^{T} \mathbb{E}\left[G\left(\theta_{t}, \mathbf{C}_{t}, \mathbf{p}_{t}\right) \mid \theta_{1}=\theta^{\circ}, \mathbf{C}_{1}=\mathbf{c}^{\circ}\right]
$$

where $\left(\theta^{\circ}, \mathbf{c}^{\circ}\right)$ is the initial condition, and $G\left(\theta_{t}, \mathbf{C}_{t}, \mathbf{p}_{t}\right)$ is the per-stage cost function defined as

$$
G\left(\theta_{t}, \mathbf{C}_{t}=\mathbf{u}, \mathbf{p}_{t}=\mathbf{v}\right)=\phi\left(\theta_{t}\right)+\beta \mathcal{Q}(\mathbf{u}, \mathbf{v})
$$

where the weighting factor $\beta \geq 0$ is a trade-off parameter which assumes the role of Lagrangian multiplier and specifies the relative importance of total power consumption across the sensors over the expected estimation error.

In fact, the average cost MDP problem (15) has a composite state space $\mathcal{T}_{\theta} \times \mathcal{C}^{M}$ and action space $\mathbb{R}^{M} \times \mathcal{P}^{M}$ with $(\theta, \mathbf{C})$, and $(\mathbf{r}, \mathbf{p})$ being the state and action respectively. In order to devise a numerical solution to (15), we discretize 
the continuum information state $\theta$. We use the discretization procedure presented in [5]. We choose a step size $\frac{1}{N}$ and approximate the continuum information state $\theta \in \mathcal{T}_{\theta}$ by discretized values $\vartheta \in \mathcal{T}$, where $\mathcal{T} \subset \mathcal{T}_{\theta}$ is the state space of the discretized information state. Note that after discretization, we still have that $\|\vartheta\|_{1}=1, \forall \vartheta \in \mathcal{T}$. In further analysis for numerical tractability, we consider a variant of the above problem (15) by restricting the range space for the variable $r$ to a finite set of discrete values in $\mathbb{R}^{M}$. Let the range space of $r_{m, t}$ be the set $\mathcal{R}=\left\{\rho_{1}, \cdots, \rho_{d}\right\}$. Hence $\mathbf{r}$ shall be chosen from the product set $\mathcal{R}^{M}$. By discretizing the information state and quantization parameter, we can now obtain a suboptimal control problem as

$$
\min _{\substack{\mathbf{r} \in \mathcal{R}^{M} \\ \mathbf{p} \in \mathcal{P}^{M}}} \limsup _{T \rightarrow \infty} \frac{1}{T} \sum_{t=1}^{T} \mathbb{E}\left[G\left(\vartheta_{t}, \mathbf{C}_{t}, \mathbf{p}_{t}\right) \mid \vartheta_{1}=\vartheta^{\circ}, \mathbf{C}_{1}=\mathbf{c}^{\circ}\right]
$$

where $\vartheta_{t} \in \mathcal{T}$ represents a discretized approximation of $\theta_{t}$, and $\left(\vartheta^{\circ}, \mathbf{c}^{\circ}\right)$ is the initial state.

The optimization problem (17) is, indeed, an average cost MDP problem with a finite state space $\mathcal{S}=\mathcal{T} \times \mathcal{C}^{M}$ and a finite action space $\mathcal{A}=\mathcal{R}^{M} \times \mathcal{P}^{M}$. The Bellman optimality equation associated with the problem (17) is given by

$$
\begin{aligned}
& \lambda+h(\mathbf{c}, \vartheta) \min _{\substack{\mathbf{r} \in \mathcal{R}^{M} \\
\mathbf{p} \in \mathcal{P}^{M}}}\left[G(\vartheta, \mathbf{c}, \mathbf{p})+\sum_{\mathbf{y}^{f}, \mathbf{c}^{\prime}} p\left(\mathbf{c}^{\prime} \mid \mathbf{c}\right)\left\|\boldsymbol{\Lambda}\left(\mathbf{r}, \mathbf{p}, \mathbf{y}^{f}, \mathbf{c}^{\prime}\right) \vartheta\right\|_{1} \times\right. \\
&\left.h\left(\mathbf{c}^{\prime},\left[\frac{\boldsymbol{\Lambda}\left(\mathbf{r}, \mathbf{p}, \mathbf{y}^{f}, \mathbf{c}^{\prime}\right) \vartheta}{\left\|\boldsymbol{\Lambda}\left(\mathbf{r}, \mathbf{p}, \mathbf{y}^{f}, \mathbf{c}^{\prime}\right) \vartheta\right\|_{1}}\right]_{\text {round }}\right)\right]
\end{aligned}
$$

where [.] round $: \mathcal{T}_{\theta} \mapsto \mathcal{T}$ is the discretization operator for the information state as described in [5], $\lambda$ is the gain or average cost per period in steady state which we are after its optimal value $\lambda^{*}$, and $h$ is the differential cost.

Assume that the quantization and power level parameters $\left(\mathbf{r}_{t}, \mathbf{p}_{t}\right)$ at each time instance $t$ are specified by a Markov stationary policy $\mu^{\infty}=\{\mu, \mu, \cdots\}$ which specifies $\left(\mathbf{r}_{t}, \mathbf{p}_{t}\right)$ as a function of the discretized information state $\vartheta_{t-1}$ and the channel state $\mathbf{C}_{t-1}$. Let $\Pi$ denote the set of all Markov stationary policies $\mu^{\infty}$. Before establishing solvability of the above Bellman equations, we need to introduce the following assumption:

(A1) For any $\mathbf{r} \in \mathcal{R}^{M}, \mathbf{p} \in \mathcal{P}^{M}, \mathbf{y}^{f} \in\left\{a_{1}, a_{2}\right\}^{M}$, and $\mathbf{c} \in \mathcal{C}^{M}$, the matrix $\boldsymbol{\Lambda}\left(\mathbf{r}, \mathbf{p}, \mathbf{y}^{f}, \mathbf{c}\right)$ is non-singular and strictly positive.

The following theorem establishes the existence of solutions to the Bellman equation (18).

Theorem 3.1: Under assumption (A1), there exists a scalar $\lambda \in \mathbb{R}$, and a bounded function $h: \mathcal{C}^{M} \times \mathcal{T} \mapsto \mathbb{R}$ which satisfies the Bellman equation (18).

Remark: Note that Theorem 3.1 holds under the fact that the state space $\mathcal{S}$ and the action space $\mathcal{A}$ are both finite, and the per-stage cost $G$ is bounded, that is, $|G(\vartheta(\mathbf{r}, \mathbf{p}), \mathbf{c}, \mathbf{p})| \leq K<\infty$ for $\forall \mathbf{c} \in \mathcal{C}^{M}, \vartheta \in \mathcal{T}, \mathbf{r} \in \mathcal{R}^{M}$, $\mathbf{p} \in \mathcal{P}^{M}$. The proof is excluded as it is very similar to the result presented in [5].

The optimality equation (18) can be solved via dynamic programming techniques. In order to find the solution to the optimality equation (18), we apply a relative value iteration algorithm. Under assumption (A1), this algorithm finds a deterministic stationary $\epsilon$-optimal policy $\left(\mu_{\epsilon}^{*}\right)^{\infty}$ and an approximation to its gain as the value of the optimal cost $\lambda^{*}$.

\section{Optimizing The Set of QuANTIZATION THRESHOLDS}

In this section, we consider the problem of finding the optimal set of quantization thresholds $\mathcal{R}=\left\{\rho_{1}, \cdots, \rho_{d}\right\}$, to be used in the optimization problem (17) as an input parameter. We consider the dynamic quantization system as a scalar-valued map function $\mathcal{L}: \mathbb{R}^{d} \mapsto \mathbb{R}_{+}$in which we have $\lambda=\mathcal{L}\left(\eta ; \beta^{\circ}\right)$, where $\lambda$ is the value of $\epsilon$-optimal cost found by the relative value iteration algorithm for a given set of quantization thresholds $\eta$. Here, $\eta$ may be viewed as a vector in $\mathbb{R}^{d}$, where $d$ is the cardinality of the set $\mathcal{R}$. Thus, the problem of finding the optimal set $\mathcal{R}^{*}$ can be translated into finding $\eta^{*}$ which minimizes the objective function $\mathcal{L}($.) over feasible values of $\eta$, that is,

$$
\eta^{*}=\arg \min _{\eta \in \mathbb{R}^{d}}\{\lambda=\mathcal{L}(\eta ; \beta)\}, \quad \lambda, \beta \in \mathbb{R}_{+} .
$$

The optimization problem (19) can be viewed as the classical problem of stochastic optimization of a multivariate system. Note that for the quantization problem at hand, the $\epsilon$ optimal cost $\lambda$ in the optimization problem (19) represents a noisy measurement of the objective function $\mathcal{L}($.$) . Moreover,$ in our problem, no direct measurements (with or without noise) of the gradient of the objective function are assumed available. Thus, we are looking for a gradient-free stochastic optimization algorithm which approximates the gradient $g(\eta)$ only from the measurements of the objective function $\mathcal{L}($.). The method we apply in this problem is an enhanced version of the simultaneous perturbation stochastic approximation (SPSA) method introduced in [13]. We apply the enhanced version of the SPSA algorithm called adaptive stochastic approximation (ASP) studied in [14]. The ASP algorithm iteration starts from an initial estimate of the optimal $\eta$. In order to find the initial estimate $\hat{\eta}_{0}$, we use a vector quantizer algorithm called LBG algorithm introduced in [15].

\section{Simulation Results}

In this section, we present some numerical examples illustrating the performance trade-off achieved by the dynamic quantization algorithm between the average estimation error and the total average power consumption. The simulations are performed for two different Markov random processes $\left\{X_{t}, t \geq 1\right\}$ and under various fading statistics, wireless channel noise, and measurement noise of the sensors. The optimal policies are obtained using the unconstrained MDP formulation (17). We use the relative value iteration algorithm in order to obtain the optimal deterministic policies and the optimal cost. Throughout the following experiments, some of the parameters are assumed fixed as following unless otherwise mentioned: the step size $1 / N$ in discretization of the information state $\theta$ is chosen $1 / N=0.01 ; \kappa$, the path loss exponent of the wireless channel, is considered $\kappa=2$ for the deployment of the sensors in an open rural area; the constant coefficient $\alpha$ for computing crossover probabilities is taken $\alpha=1$.

\section{A. State Estimation of a Two-State Markov Chain}

In this section, we examine state estimation of a two-state Markov chain $\left\{X_{t}, t \geq 1\right\}$ using two sensors. The sensors are located in different distances from the fusion centre with distance vector $d=[100.0,200.0]^{T}$, where the distance figures are given in meters. For the Markov chain $\left\{X_{t}\right\}$, the state 


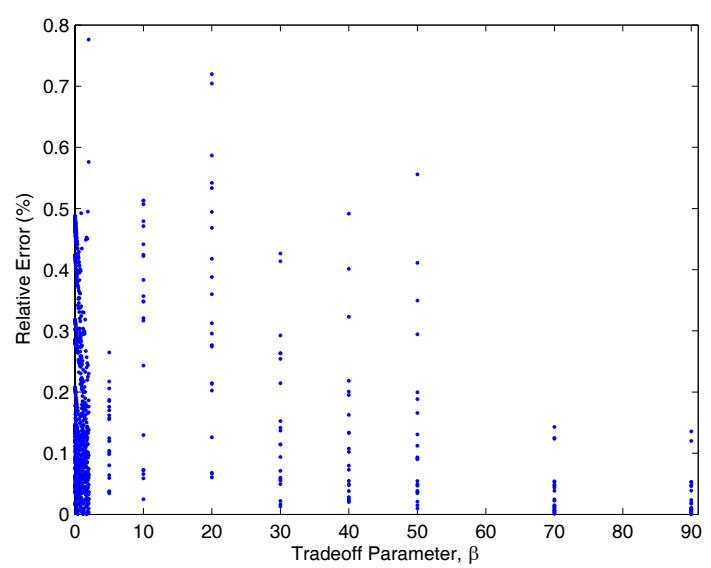

Fig. 1. Relative error between $\epsilon$-optimal cost found by relative value iteration algorithm and the cost computed by simulation.

space is $\mathcal{X}=\left\{s_{1}=0, s_{2}=2.5\right\}$ and the transition probability matrix is given by

$$
\mathbf{P}=\left[\begin{array}{ll}
0.8 & 0.2 \\
0.4 & 0.6
\end{array}\right]
$$

The measurement noise of the sensors are assumed to be i.i.d. and normally distributed with zero mean and identical variance $\sigma^{2}=0.3$, that is, $\mathcal{V}_{m, t} \sim \mathcal{N}(0,0.3)$ for $m=1,2$. The wireless flat fading channels from the sensors to the fusion centre are assumed to be independent and each channel is modeled by a two state Markov chain with state space $\mathcal{C}=\left\{c_{1}, c_{2}\right\}$. We consider asymmetric channels for the two sensors, that is, the channels have different fading statistics given by the following transition probability matrices

$$
\mathbf{P}_{1}^{\mathcal{C}}=\left[\begin{array}{ll}
0.85 & 0.15 \\
0.25 & 0.75
\end{array}\right], \mathbf{P}_{2}^{\mathcal{C}}=\left[\begin{array}{cc}
0.6 & 0.4 \\
0.55 & 0.45
\end{array}\right] .
$$

The channel states $c_{1}$ and $c_{2}$ represent the corresponding channel gains $g_{1}=10^{-10}$ and $g_{2}=10^{-11}$ respectively. The proportional magnitude of the channel gain $g_{1}$ relative to $g_{2}$ indicates $10 d B$ drop in signal-to-noise ratio $\gamma$ when the wireless channel deteriorates from state $c_{1}$ to $c_{2}$ and the power remains unchanged. The noise power of the wireless channel for every sensor is $\sigma_{\nu}^{2}=10^{-16} \mathrm{~W}$. The action space of the power level parameter for every sensor is considered as $\mathcal{P}=\{60,40,20,5\}$, where the power levels are given in $m W$. However, it is worth mentioning that these power levels are chosen as typical values, and the devised algorithm can be used for any technology-specific values of power levels. Using the given transition matrix for each channel Markov chain, the steady-state distribution of the channel states for the two sensors can be calculated as $\pi^{1}=[0.625,0.375]^{T}$ and $\pi^{2} \approx[0.579,0.421]^{T}$ which are used in computing the power budget function $\mathcal{Q}\left(\mathbf{C}_{t}, \mathbf{p}_{t}\right)$.

In order to study the effect of the measurement noise variance $\sigma^{2}$ on the optimal error/power curve, we use Monte Carlo simulations to compute the long term average of both state estimation error and power for the $\epsilon$-optimal policy $\mu^{*}$ found by the relative value iteration algorithm. We compute the estimation error and power consumption using a sample path $\omega$ consisting of a sequence of the received measurements $\left\{\mathbf{Y}_{t}^{f}(\omega)\right\}_{t=1}^{T}$, and a sequence of the channel states $\left\{\mathbf{C}_{t}(\omega)\right\}_{t=1}^{T}$ for $T=50 \times 10^{3}$. Note that the sequence

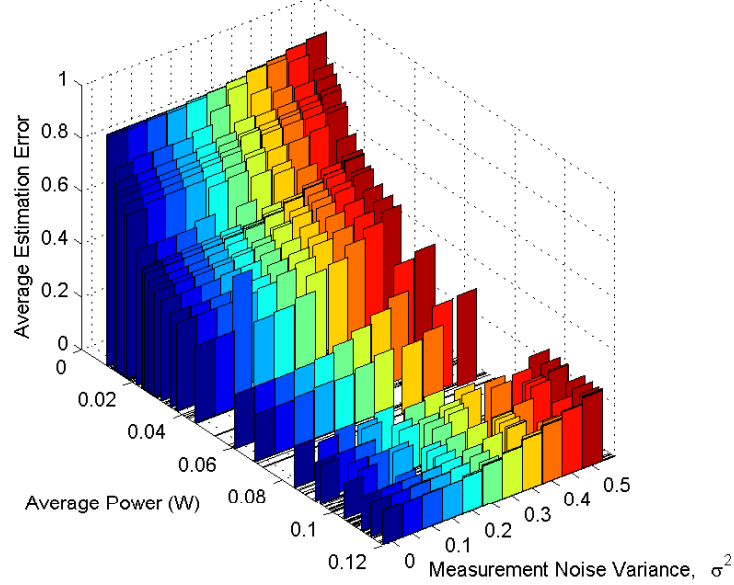

Fig. 2. Optimal Error/Power curves for different values of the measurement noise.

$\left\{\mathbf{Y}_{t}^{f}(\omega)\right\}_{t=1}^{T}$ is, in fact, determined by the corresponding sequences of the measurement noise $\left\{\mathbf{V}_{t}(\omega)\right\}_{t=1}^{T}$ and Markov chain state $\left\{X_{t}(\omega)\right\}_{t=1}^{T}$ for a given sample path $\omega$. Let $\left\{\theta_{t}(\omega)\right\}_{t=1}^{T}$ be a sequence of the information state computed using recursion (10) at time steps $t \geq 2$ having two sequences $\left\{\mathbf{Y}_{t}^{f}(\omega)\right\}$ and $\left\{\mathbf{C}_{t}(\omega)\right\}$. Based on the sequence $\left\{\theta_{t}(\omega)\right\}$ and the discretization operator for the information state, we obtain the sequence $\left\{\vartheta_{t}(\omega)\right\}_{t=1}^{T}$ for a given sample path $\omega$. The optimal action sequence $\left\{\mathcal{A}_{t}(\omega)\right\}_{t=2}^{T}$, where $\mathcal{A}_{t}=\left(\mathbf{r}_{t}, \mathbf{p}_{t}\right)$, is then determined by the optimal policy $\mu^{*}$. Then, the average estimation error and average power are computed by averaging on 30 runs of the simulation. Fig. 1 illustrates concurrence of the $\epsilon$-optimal per-stage cost computed by relative value iteration algorithm and the cost computed by simulation for 945 experiments in which each experiment has been run with different values of the parameters $\sigma^{2}, \sigma_{\nu}^{2}$, and $\mathcal{R}$. As it can be seen in Fig. 1, the relative error is upper bounded by $1 \%$ and for $98.8 \%$ of the experiments, the relative error is less than half a percent.

Fig. 2 illustrates the optimal estimation error/total power tradeoff curves for various values of measurement noise variance in the range of $0 \leq \sigma^{2} \leq 0.5$. For each value of the tradeoff parameter $\beta$ along every error/power curve, the optimal set of quantization thresholds $\mathcal{R}^{*}$ with cardinality $d=6$ has been found using the gradient-free stochastic optimization method ASP. In Fig. 2, for the same average power, a trend of consistent decrease of the estimation error with the decrease of the measurement noise variance can be observed. However, the relative decrease rate is much higher for the power levels along the tail of the curve where the value of the trade-off parameter $\beta$ is smaller (i.e., when $\beta \rightarrow 0$ ). The reason for this is that the contribution of the estimation error in the cost function $G($.$) is$ quite considerable when $\beta \rightarrow 0$. Moreover, in order to achieve the same estimation error, it requires larger transmission power in sensor nodes for higher values of the measurement noise than lower ones. This is because higher power level results in higher received SNR $\gamma$ at the fusion centre which then leads to a lower crossover probability that compensates for the highly noisy sensors' observations in equation (8). For the trivial case $\sigma^{2}=0$, the quantizer output at each sensor node is determined directly from the state value $x_{t} \in \mathcal{X}$ of the Markov chain $\left\{X_{t}, t \geq 1\right\}$. 


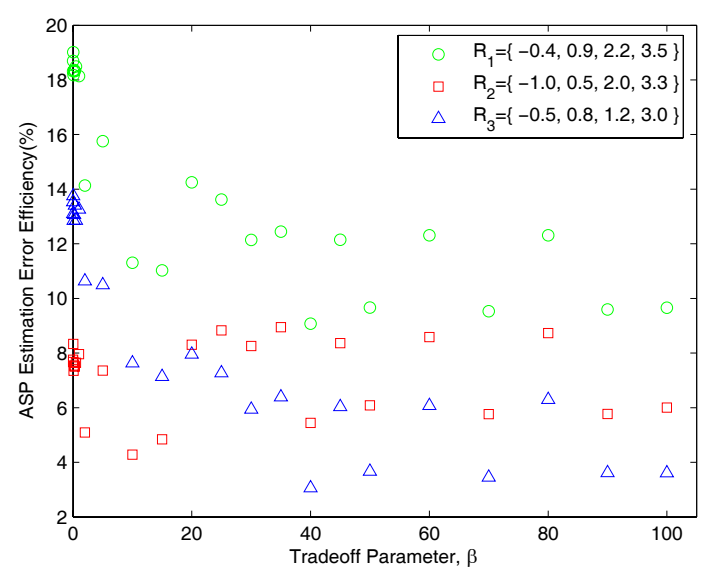

Fig. 3. The improvement in state estimation error achieved by using the ASP result set $\mathcal{R}^{*}$ over against some arbitrary sets for different values of trade-off parameter $\beta$.

\section{B. ASP Results for State Estimation of a Three-State Markov Chain via Two Sensors}

The following results provide the improvement, in sense of state estimation error, achieved by using ASP optimization method in state estimation of a three-state Markov chain $\left\{X_{t}, t \geq 1\right\}$ via two sensors. For the Markov chain $\left\{X_{t}\right\}$, the state space is $\mathcal{X}=\left\{s_{1}=0, s_{2}=1, s_{3}=2.5\right\}$ and the transition probability matrix is given by

$$
\mathbf{P}=\left[\begin{array}{ccc}
0.9 & 0.1 & 0 \\
0.1 & 0.8 & 0.1 \\
0 & 0.15 & 0.85
\end{array}\right]
$$

We consider symmetric channels for the two sensors with equal transition probability matrix $\mathbf{P}_{1}^{\mathcal{C}}$ given in $\mathrm{Sec}$ tion V-A.The transmission power level for every sensor is optimized from the set $\mathcal{P}=\{60,30,10\}$, with the power values being in $m W$. The quantization threshold parameter for every sensor is optimized from the set $\mathcal{R}=\left\{\rho_{1}, \cdots, \rho_{d}\right\}$ with cardinality $d=4$. The other input parameters are the same as those mentioned in Section V-A.

Fig. 3 illustrates the improvement in state estimation error achieved by using the ASP result set $\mathcal{R}^{*}$ versus estimation error obtained from some arbitrary sets. The first set is given by $\mathcal{R}_{1}=\{-0.4,0.9,2.2,3.5\}$ which contains equally-spaced threshold values starting from -0.4 with step size 1.3 . The set $\mathcal{R}_{1}$ is covering the most probable deviation ${ }^{1}$ from the third Markov chain state value $s_{3}=2.5$. As specified by circle markers in Fig. 3, for different values of the tradeoff parameter $\beta$, the state estimation error obtained from the ASP result set $\mathcal{R}^{*}(\beta)$ comparing to the one achieved by using the set $\mathcal{R}_{1}$ shows $9.08-19.01 \%$ improvement with average improvement of $14.02 \%$. For the second set $\mathcal{R}_{2}=\{-1.0,0.5,2.0,3.3\}$, the error improvement, specified by square markers in Fig. 3, lies in the range $4.28-8.95 \%$ with the average of $7.19 \%$. The set $\mathcal{R}_{2}$ is covering the most probable deviation from the first Markov chain state value $s_{1}=0.0$. As for the third set $\mathcal{R}_{3}=\{-0.5,0.8,1.2,3.0\}$, specified by triangle markers in Fig. 3, the ASP result set $\mathcal{R}^{*}$ shows $3.06-13.75 \%$ better error efficiency with a mean efficiency of $8.55 \%$. The set $\mathcal{R}_{3}$ is

${ }^{1}$ that is, $\pm 2 \sigma$ which accounts for up to $95.5 \%$ of the values for random variable $Y_{m, t}$ drawn from Gaussian distribution $\mathcal{N}\left(s_{3}, \sigma^{2}\right)$ when Markov chain state is $X_{t}=s_{3}=2.5$. chosen such that two of the threshold values are concentrated around the Markov chain mid-state $s_{2}=1.0$ and the other two thresholds are evaluated with mid-points in the most probable deviation range from the Markov chain state $s_{1}$ and $s_{3}$.

\section{CONCLUding REMARKS}

In this paper, we designed a novel optimal dynamic quantizer design algorithm with optimal power allocation for state estimation of hidden Markov models using multiple sensors communicating with a fusion centre over temporally correlated flat fading channels. We formulated the problem as a stochastic control problem with a Markov decision process and used dynamic programming techniques to find the optimal cost and the optimal deterministic stationary policy which adapts the values for the quantization thresholds and power levels based on the state estimate and channel state information available at the fusion centre. Moreover, we employed a gradient-free stochastic optimization technique to determine the optimal set of quantization thresholds for a given value of the tradeoff parameter. Simulation studies were carried out to illustrate the optimal average estimation error/average total power tradeoff performance under various channel fading, noise and senor measurement noise parameter values.

\section{REFERENCES}

[1] M. D. Bisceglie and M. Longo, "Decentralized encoding of a remote source," ACM Signal Processing, vol. 55, no. 1, pp. 15-29, November 1996.

[2] W.-M. Lam and A. Reibman, "Design of quantizers for decentralized estimation systems," IEEE Transactions on Communications, vol. 41 no. 11, pp. 1602-1605, November 1993.

[3] J.-F. Chamberland and V. V. Veeravalli, "Decentralized detection in sensor networks," IEEE Transactions on Signal Processing, vol. 51, no. 2, pp. 407-416, February 2003.

[4] J.-S. Leu and A. Papamarcou, "Asymptotic optimality of likelihood ratio threshold tests in decentralized detection," IEEE Transactions on Information Theory, vol. 45, pp. 572-585, March 1999.

[5] M. Huang and S. Dey, "Dynamic quantizer design for hidden markov state estimation via multiple sensors with fusion center feedback," IEEE Transactions on Signal Processing, vol. 54, no. 8, pp. 2887-2896, August 2006.

[6] H. C. Papadopoulos, G. W. Wornell, and A. V. Oppenheim, "Sequential signal encoding from noisy measurements using quantizers with dynamic bias control," IEEE Transactions on Information Theory, vol. 47, no. 3, pp. 978-1002, March 2001.

[7] Z.-Q. Luo and J.-J. Xiao, "Decentralized estimation in an inhomoge neous sensing environment," IEEE Transactions on Information Theory, vol. 51, no. 10, pp. 3564-3575, October 2005.

[8] A. Ribeiro and G. Giannakis, "Bandwidth-constrained distributed estimation for wireless sensor networks-part i: Gaussian case," IEEE Transactions on Signal Processing, vol. 54, no. 3, pp. 1131-1143, March 2006.

[9] J.-J. Xiao, S. Cui, Z.-Q. Luo, and A. J. Goldsmith, "Power scheduling of universal decentralized estimation in sensor networks,", in IEEE Transactions on Signal Processing, vol. 52, no. 2, February 2006, pp. $413-422$

[10] J.-F. Chamberland and V. V. Veeravalli, "The impact of fading on decentralized detection in power constrained wireless sensor networks," in Proceedings of the IEEE International Conference on Acoustics, Speech, and Signal Processing, vol. 3, Montreal, Canada, May 2004, pp. $837-840$.

[11] M. Huang and S. Dey, "Dynamic quantization for multisensor estimation over bandlimited fading channels" IEEE Transactions on Signal Processing, vol. 55, no. 9, pp. 4696-4702, September 2007.

[12] X. Luo and G. B. Giannakis, "Energy-constrained optimal quantization for wireless sensor networks," in Proceedings of the first IEEE Communications Society Conference on Sensor and Ad Hoc Communication and Networks, CA, USA, October 2004, pp. 272-278.

[13] J. C. Spall, "Multivariate stochastic approximation using a simultaneous perturbation gradient approximation," IEEE Transactions on Automatic Control, vol. 37, no. 3, pp. 332-341, March 1992.

[14] _ - "Adaptive stochastic approximation by the simultaneous perturbation method," IEEE Transactions on Automatic Control, vol. 45, no. 10, pp. 1839-1853, October 2000.

[15] Y. Linde, A. Buzo, and R. M. Gray, "An algorithm for vector quantize design," IEEE Transactions on Communications, vol. 28, no. 1, pp. 84 95, January 1980. 